\title{
Silver Nanoparticles Capped with $p$-Hydroxybenzoic Acid as a Colorimetric Sensor for the Determination of Paraquat
}

\author{
Gusrizal Gusrizall, $^{1,}$, Sri Juari Santosa ${ }^{2}$, Eko Sri Kunarti ${ }^{2}$, and Bambang Rusdiarso ${ }^{2}$ \\ ${ }^{1}$ Department of Chemistry, Faculty of Mathematics and Natural Sciences, Universitas Tanjungpura, \\ Jl. Prof. Dr. H. Hadari Nawawi, Pontianak 78124, Indonesia \\ ${ }^{2}$ Department of Chemistry, Faculty of Mathematics and Natural Sciences, Universitas Gadjah Mada, \\ Sekip Utara, Yogyakarta 55281, Indonesia
}

${ }^{*}$ Corresponding author:

email: gusrizal@chemistry.untan.ac.id

Received: August 16, 2019

Accepted: October 1, 2019

DOI: $10.22146 /$ ijc. 48806

\begin{abstract}
Highly stable silver nanoparticles capped with p-hydroxybenzoic acid were synthesized by reducing silver ion with $\mathrm{p}$-hydroxybenzoic acid and used for the detection of paraquat. The synthesized silver nanoparticles, which are yellow, exhibited an absorption peak at $420 \mathrm{~nm}$ when measured with a UV-visible spectrophotometer due to the surface plasmon resonance. In the presence of paraquat, the color of silver nanoparticles changed from yellow to purple accompanied by the appearance of a new peak at $580 \mathrm{~nm}$ in addition to the peak at $420 \mathrm{~nm}$. In order to obtain optimum experimental conditions, temperature, and time of reaction were optimized, and the ratio of absorbance obtained at $580 \mathrm{~nm}$ and $420 \mathrm{~nm}\left(A_{580} / A_{420}\right)$ were monitored. The $A_{580} / A_{420}$ is proportional to the concentration of paraquat. Under the most favorable condition, the calibration curve showed a high level of linearity ranging from $6.0 \times 10^{-4}$ to $1.0 \times 10^{-3} \mathrm{M}$, and the detection limit was found to be $8.30 \times 10^{-6} \mathrm{M}$. Silver nanoparticles capped with $\mathrm{p}$-hydroxybenzoic acid was found to be useful for the colorimetric determination of paraquat in the aqueous medium.
\end{abstract}

Keywords: nanoparticles; paraquat; p-hydroxybenzoic acid; sensor; silver

\section{- INTRODUCTION}

Over the past few years, scientific publications have shown a significant increase in research on the exploration of nanoparticles. Among the metal nanoparticles, silver nanoparticles have attracted significant interest. Silver nanoparticles have been familiar as an antibacterial agent [1]. In addition to being an antibacterial agent, silver nanoparticles have been used in the development of analytical methods.

There is an increase in research using silver nanoparticles as a colorimetric sensor. The basis of measurement is a change in surface plasmon resonance spectra of silver nanoparticles caused by the aggregation of nanoparticles which results from the interaction of an analyte with the capping agent molecules covering the surface of the nanoparticles [2-3].

Surface plasmon resonance is an optical property of metal nanoparticles originating from the interaction of light with the mobile conduction electron of the metal and is spectrally observed as a maximum absorbance in the visible range. Silver and gold nanoparticles display different positions of absorbance maxima, e.g., at the wavelength of 415 and $525 \mathrm{~nm}$, respectively [4]. In addition to the type of metal, the position of absorbance maxima is dependent on the size of particles that could be controlled by the reducing and capping agent used in the synthesis [5-6].

Many methods for the synthesis of silver nanoparticles are available. In the chemical reduction method, the precursor of silver, the reducing agent, and the stabilizer or capping agent are needed [7]. Sodium borohydride and sodium citrate are usually used in the reduction of silver ions to produce silver nanoparticles, and polymers such as polyvinyl alcohol stabilize the silver nanoparticles during and after the formation of nanoparticles [5]. 
Silver nanoparticles were used as colorimetric sensors for metal ions such as $\mathrm{Cu}(\mathrm{II})$ [8], $\mathrm{Cr}(\mathrm{VI})$ [2], $\mathrm{Mn}$ (II), $\mathrm{Hg}$ (II) [9], Cr(III) [10], and $\mathrm{Fe}(\mathrm{III})$ [11]. The application of silver nanoparticles as a colorimetric sensor for organic compounds is still limited. They were used for the determination of melamine [12] and herbicide sulfurazon-ethyl [13]. In this paper, silver nanoparticles capped with $p$-hydroxybenzoic acid were used for the development of a colorimetric paraquat determination.

Paraquat (1,1'-dimethyl-4-4'-bipyridinium dichloride) is one of the most widely used herbicides in agriculture. However, it is a highly toxic herbicide. The risk of Parkinson's disease has been associated with exposure to paraquat [14-15]. In considering the effect of paraquat, it is essential to monitor the existence of paraquat in the environment. Several analytical methods have been available for paraquat determination such as liquid chromatography/ electrospray ionization-mass spectrometry [16], ultraperformance liquid chromatography-mass spectrometry/ mass spectrometry (UPLC-MS/MS) [17]. Because of the high cost of the equipment for determination, it is necessary to replace it with a rapid and straightforward method.

Previously, we have successfully synthesized highly stable silver nanoparticles capped with $p$-hydroxybenzoic acid [18], however their application has not been explored. As presented in this paper, we have developed an analytical method for paraquat determination using silver nanoparticles capped with $p$-hydroxybenzoic acid. The data presented in this paper is taken from the doctoral dissertation submitted to the Department of Chemistry Universitas Gadjah Mada by one of the authors [19].

It is predicted that paraquat initiates the aggregation of silver nanoparticles capped with $p$-hydroxybenzoic acid. Paraquat interacts with the $p$-hydroxybenzoic acid covering the silver nanoparticles through the mechanism of ion-pair as well as the formation of charge transfer complex. Paraquat acts as a bridge between the nanoparticles and causes the distance between the particles to become closer and produces a change in surface plasmon resonance spectra. The change of surface plasmon resonance spectra is estimated to be proportional to the concentration of paraquat. Therefore, silver nanoparticles capped with $p$-hydroxybenzoic acid was used for the quantitative determination of paraquat.

\section{- EXPERIMENTAL SECTION}

\section{Materials}

Sodium hydroxide (Merck), silver nitrate (Merck), potassium chloride (Merck), ammonium chloride (Merck), disodium hydrogen phosphate (Merck), $p$ hydroxybenzoic acid (Sigma-Aldrich), and paraquat (Sigma-Aldrich) were used as received without any further purification. Throughout the experiment, we used double distilled water.

\section{Instrumentation}

UV-visible spectrophotometer (Shimadzu UV-1700 PharmaSpec) was used to obtain the surface plasmon resonance spectra, and the transmission electron microscope (JEOL JEM-1400) was used to determine the size and morphology of the silver nanoparticles.

\section{Procedure}

\section{Synthesis of silver nanoparticles}

Silver nanoparticles capped with $p$ hydroxybenzoic acid were synthesized through the chemical reduction of silver nitrate with $p$ hydroxybenzoic acid as a reducing agent without additional capping agents in an aqueous medium [20]. A 5 - $\mathrm{mL}$ of $2.0 \times 10^{-4} \mathrm{M}$ silver nitrate were added to $5 \mathrm{~mL}$ pH 11-adjusted $4.0 \times 10^{-3} \mathrm{M} p$-hydroxybenzoic acid and was then heated in a boiling water bath for $1 \mathrm{~h}$. A UVvisible spectrophotometer and a transmission electron microscope were used to characterize the synthesized silver nanoparticles.

\section{Colorimetric detection of paraquat}

The procedure for the colorimetric detection of paraquat is as follows: $1 \mathrm{~mL}$ of paraquat standard was introduced to $2 \mathrm{~mL}$ of synthesized silver nanoparticles and heated in a boiling water bath for $10 \mathrm{~min}$. When the reaction was complete, the mixture was then cooled in tap water. The color of the solution changed from yellow to purple, and the change of color and absorbance intensity was monitored using a UV-visible 
spectrophotometer. The performance of silver nanoparticles capped with $p$-hydroxybenzoic acid in the detection of paraquat was evaluated.

\section{- RESULTS AND DISCUSSION}

\section{Synthesis of Silver Nanoparticles}

The formation of silver nanoparticles was visible because of the appearance of the yellow color in the mixtures of reaction. The color was due to the surface plasmon resonance and confirmed by measurement with a UV-visible spectrophotometer. The UV-visible spectra of silver nanoparticles synthesized by the reduction of silver nitrate with $p$-hydroxybenzoic acid are shown in Fig. 1(a). The spectra exhibited absorbance maxima at a wavelength of $420 \mathrm{~nm}$. The transmission electron microscope image, as shown in Fig. 2, proves the formation of silver nanoparticles. In a previous paper, we reported that the reduction of silver nitrate with $p$-hydroxybenzoic acid without additional capping agents produced silver nanoparticles capped with $p$-hydroxybenzoic acid with an average size of $26 \pm 11 \mathrm{~nm}$ [20].

The size of the particles and the position of the peak maxima at the surface plasmon resonance spectra of the silver nanoparticles varies with reducing and capping agents used in the synthesis. The reduction of the silver ion with glucose using polyvinylpyrrolidone as a capping agent produces silver nanoparticles with a peak at $420 \mathrm{~nm}$ and a size of $50 \mathrm{~nm}$ [21]. The synthesis of silver nanoparticles using aniline and dodecylbenzene sulfonic acid as a reducing and capping agent resulted in silver nanoparticles of $10 \mathrm{~nm}$ and a peak at $410 \mathrm{~nm}$ [22]. The reducing and capping agent determines the size and shape of the silver nanoparticles, and the position of the maximum peak depends on the size and shape of the nanoparticles [23].

\section{Interaction of Silver Nanoparticles and Paraquat}

Silver nanoparticles have been used for colorimetric detection of chemical compounds. The property of silver nanoparticles used in the detection of chemical compounds is the change in surface plasmon resonance spectra due to the interaction of silver nanoparticles with an analyte. In general, surface plasmon resonance spectra

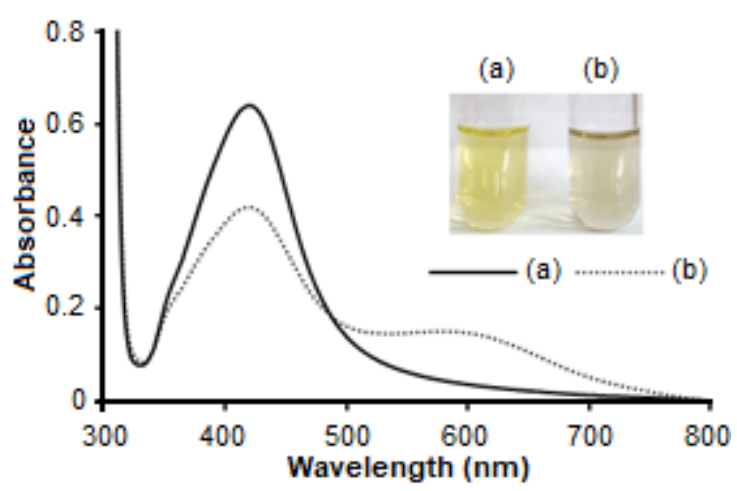

Fig 1. The surface plasmon resonance spectra of silver nanoparticles capped with $p$-hydroxybenzoic acid; (a) after synthesis and (b) after addition of paraquat

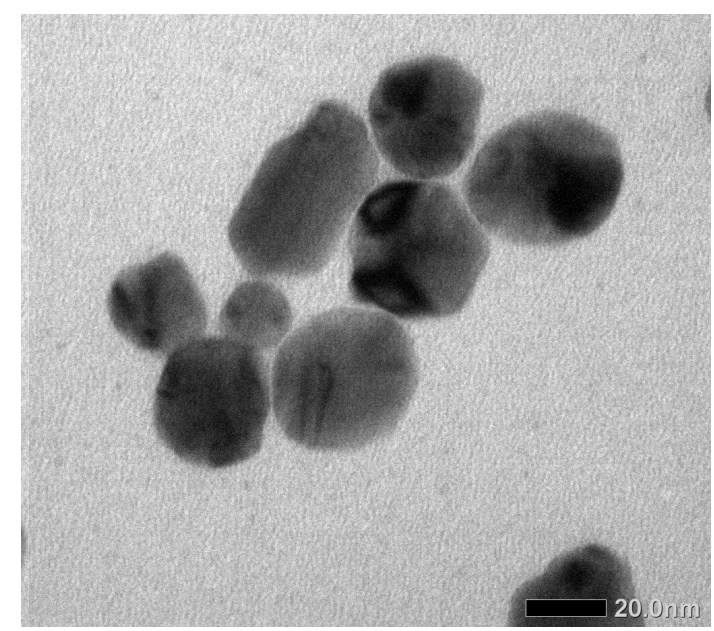

Fig 2. The TEM image of silver nanoparticles capped with $p$-hydroxybenzoic acid

of nanoparticles are sensitive to composition, size, shape, media, and the degree of aggregation of nanoparticles [24].

The addition of analytes to silver nanoparticles causes aggregation of silver nanoparticles. Surface plasmon resonance spectra of silver nanoparticles that experience aggregation are different when compared to the spectra of silver nanoparticles which do not experience aggregation. The difference that arises as a result of aggregation is the decrease in absorbance intensity at the main peak and the presence of secondary peaks at larger wavelengths. The ratio between the secondary peak and the main peak is used as a parameter for determining the amount of analyte because it represents the degree of aggregation due to the presence of the analyte [25]. 
The addition of paraquat to silver nanoparticles resulted in a change in the color of the nanoparticles from yellow to purple. This change was followed by changes in the surface plasmon resonance spectra of the nanoparticles, as shown in Fig. 1(b). The intensity of the main peak at $420 \mathrm{~nm}$ decreased, and there was a new peak at $580 \mathrm{~nm}$. The existence of secondary peaks at larger wavelengths indicates the presence of larger silver nanoparticles due to aggregation [26]. The change of spectra represented by the ratio of absorbance obtained at $580 \mathrm{~nm}$ and $420 \mathrm{~nm}$ $\left(A_{580} / A_{420}\right)$ was used as the basis for paraquat determination.

It is predicted that paraquat interacts with silver nanoparticles capped with p-hydroxybenzoic acid through the mechanism of ionic interaction between the positive charge of paraquat with the anionic group $p$ hydroxybenzoic acid covering silver nanoparticles and the formation of charge transfer complexes by $\pi-\pi$ stacking of aromatic rings. In the formation of charge transfer complexes, paraquat with high electronegativity acts as an electron acceptor, and the aromatic ring of $p$ hydroxybenzoic acid which caps silver nanoparticles acts as electron donors [27-28]. The interaction between paraquat and $p$-hydroxybenzoic acid covering silver nanoparticles places paraquat as a bridge between nanoparticles and results in a closer distance between the nanoparticles. This causes changes in the surface plasmon resonance spectra [29]. The model of interaction between paraquat and silver nanoparticles capped with $p$ hydroxybenzoic acid, which causes aggregation is proposed as shown in Fig. 3.

A similar principle in the detection of chemicals using silver nanoparticles has been applied. The binding
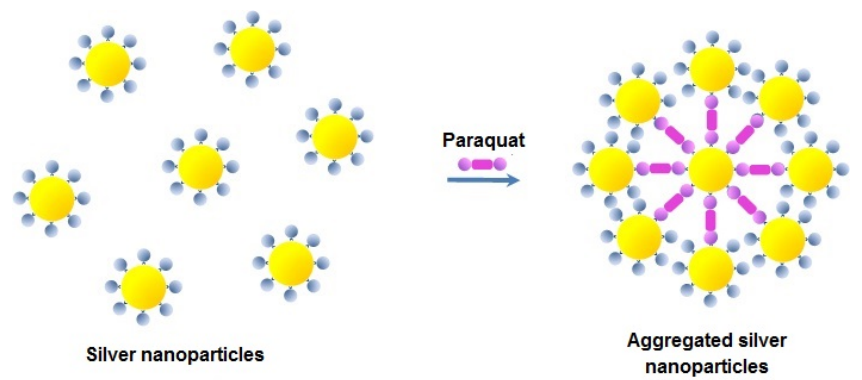

Fig 3. Model of interaction between paraquat and silver nanoparticles capped with p-hydroxybenzoic acid which causes aggregation between melamine as an analyte with dopamine functionalized the surface of silver nanoparticles and produces the aggregation of nanoparticles [12]. Interaction of $\mathrm{Cr}$ (III) with trisodium citrate that acts as a capping agent of silver nanoparticles induces the aggregation of silver nanoparticles [2]. The aggregation of silver nanoparticles causes a change in the surface plasmon resonance spectra, and the change of spectra is proportional to the concentration of the analyte.

\section{Optimization of Temperature and Reaction Time for the Reaction of Silver Nanoparticles with Paraquat}

The reaction of synthesized silver nanoparticles capped with $p$-hydroxybenzoic acid with paraquat $(1.0 \times$ $10^{-3} \mathrm{M}$ ) was performed under room temperature and heating in a boiling water bath. The reaction was monitored during a specific time by determination of $\mathrm{A}_{580} / \mathrm{A}_{420}$ to obtain the best experimental condition. Fig. 4 shows the change of surface plasmon resonance spectra of silver nanoparticles during the incubation. For the reaction under room temperature, the $A_{580} / A_{420}$ continually increases until $150 \mathrm{~min}$ of incubation. On the other hand, stable aggregate formation was faster when the reaction was kept in a boiling water bath. The data shows that temperature affected the formation of a stable aggregate. We used $10 \mathrm{~min}$ incubation in a boiling water bath as the optimum condition for the reaction of paraquat with silver nanoparticles capped with $p$ hydroxybenzoic acid.

\section{Analytical Performance of Paraquat Determination}

The analytical performance parameter such as linear range or linearity, the limit of detection, precision, and accuracy were evaluated to validate the performance of silver nanoparticles capped with $p$-hydroxybenzoic acid for the determination of paraquat. Linearity shows that the measured response is proportional to the quantity of analyte. Linearity study was performed by plotting the $\mathrm{A}_{580} / \mathrm{A}_{420}$ toward the concentration of paraquat. In this study, the concentration of paraquat used was varied from $1.0 \times 10^{-4} \mathrm{M}$ to $1.0 \times 10^{-3} \mathrm{M}$ with three replications. As shown in Fig. 5, the intensity of 

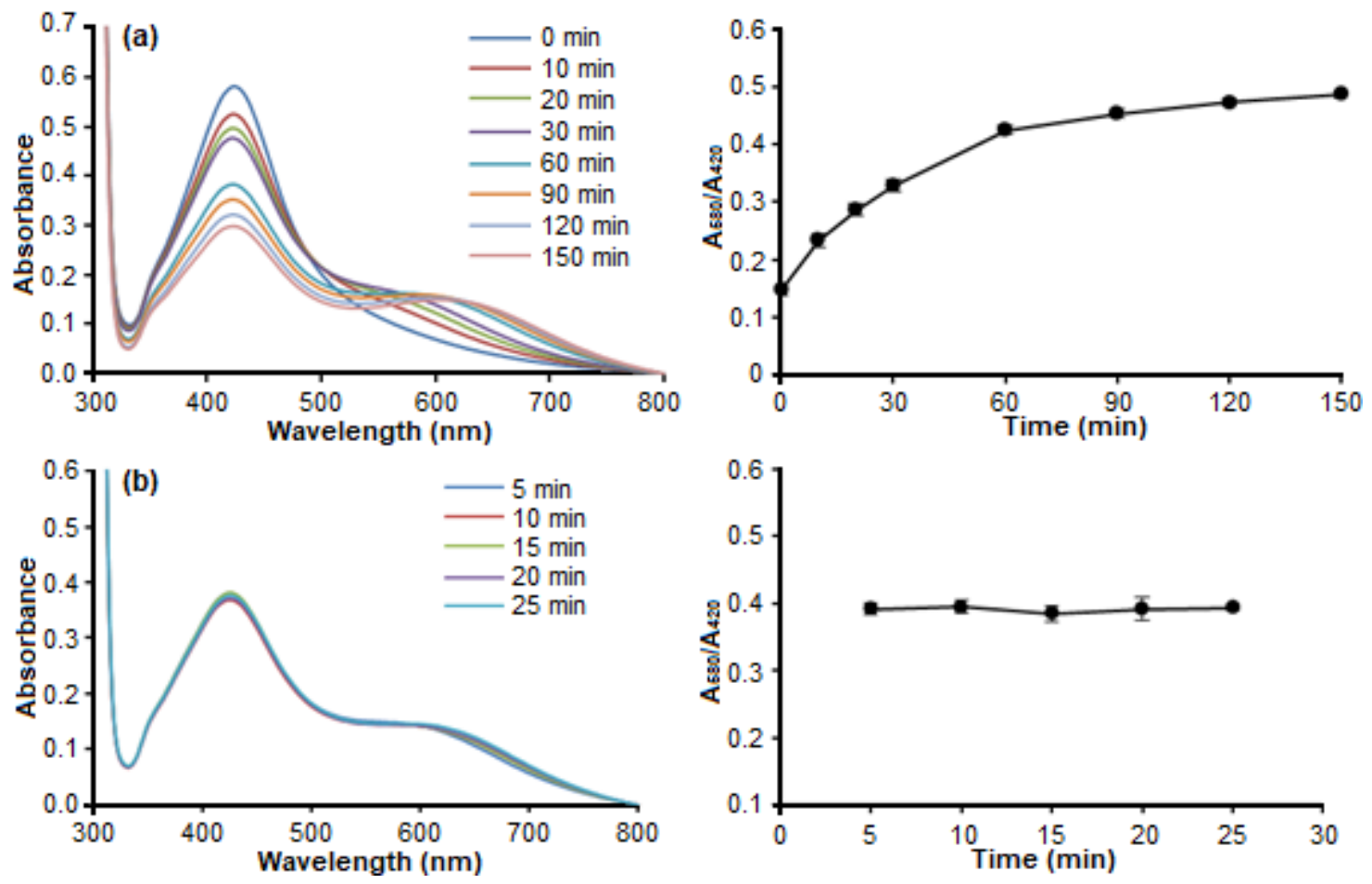

Fig 4. The change of surface plasmon resonance spectra and variation of $A_{580} / A_{420}$ of silver nanoparticles capped with $p$-hydroxybenzoic acid with the presence of paraquat during the incubation under (a) room temperature and (b) heating in boiling water bath
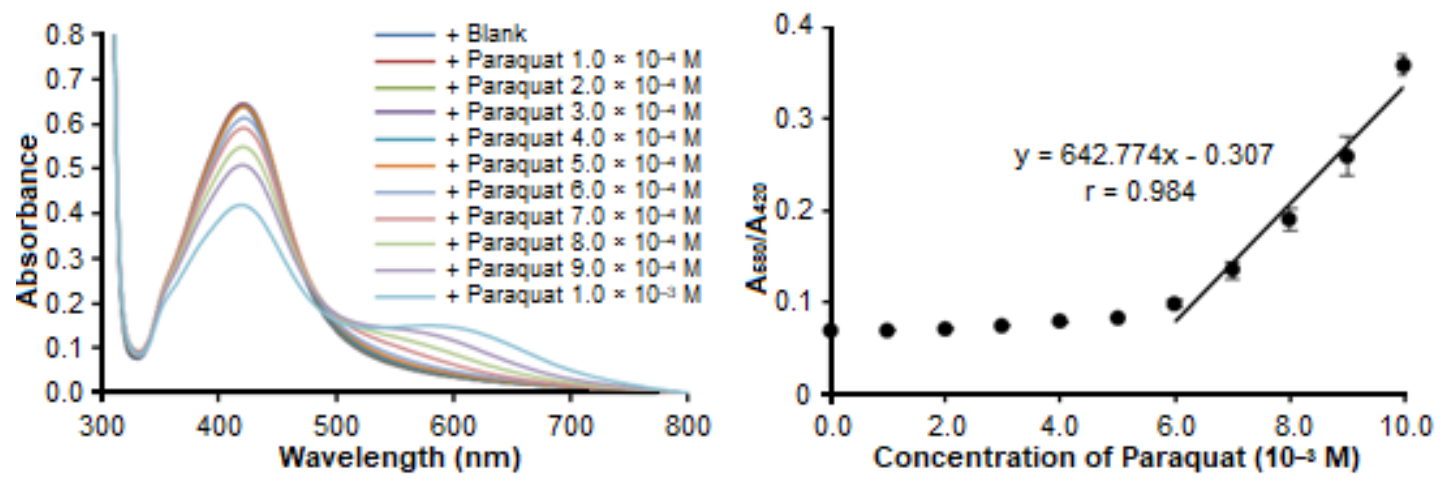

Fig 5. The surface plasmon resonance spectra of silver nanoparticles capped with $p$-hydroxybenzoic acid after the addition of paraquat with different concentration and the plot of $\mathrm{A}_{580} / \mathrm{A}_{420}$ towards the concentration of paraquat

spectra at $420 \mathrm{~nm}$ decreased gradually by the increase of paraquat concentration and followed by the increase in peak intensity at $580 \mathrm{~nm}$. The $\mathrm{A}_{580} / \mathrm{A}_{420}$ increased linearly with the concentration of paraquat ranging from $6.0 \times 10^{-4}$ to $1.0 \times 10^{-3} \mathrm{M}$ and the coefficient of regression ( $\mathrm{r}$ ) was 0.984 .

The limit of detection was evaluated by measurement of several replications of a blank solution and defined as three times the standard deviation $(\mathrm{n}=7)$. The limit of detection obtained was $8.30 \times 10^{-6} \mathrm{M}$.
The evaluation of precision was carried out by measurement of a known concentration of paraquat with several replications. The precision was represented by the coefficient of variance from seven replications of measurement. The coefficient of variance obtained was $3 \%$.

Accuracy was determined by spiking the standard solution of paraquat with the real water sample. The water sample was collected from the small canal in the 
area of the chili farm. The amount of paraquat spiked into the water sample was estimated, and then the recovery percentage was calculated. The recovery percentage for the determination of paraquat in the water sample was $121 \%$.

\section{Effect of Other Substances}

The universal inorganic and organic matrix in water from the farm area such as ammonium $\left(\mathrm{NH}_{4} \mathrm{Cl}\right)$, phosphate $\left(\mathrm{Na}_{2} \mathrm{HPO}_{4}\right)$, potassium $(\mathrm{KCl})$, and humic acid was tested to check whether they could interfere in the determination of paraquat using silver nanoparticles capped with $p$-hydroxybenzoic acid. The $\mathrm{NH}_{4} \mathrm{Cl}, \mathrm{Na}_{2} \mathrm{HPO}_{4}$, $\mathrm{KCl}$, and humic acid, individually were mixed with paraquat. The concentration of $\mathrm{NH}_{4} \mathrm{Cl}, \mathrm{Na}_{2} \mathrm{HPO}_{4}, \mathrm{KCl}$ in the mixture was $1.0 \times 10^{-3} \mathrm{M}$ and the concentration of humic acid was 1 ppm. Fig. 6 shows the interference effect of these matrices to the spectra of the mixture of silver nanoparticles and paraquat. The $\mathrm{NH}_{4} \mathrm{Cl}, \mathrm{Na}_{2} \mathrm{HPO}_{4}$, and $\mathrm{KCl}$ changed the $\mathrm{A}_{580} / \mathrm{A}_{420}$ by $1 \%, 21 \%$, and $7 \%$, respectively.

The presence of $\mathrm{Na}_{2} \mathrm{HPO}_{4}$ resulted in a significant interference effect on the $A_{580} / A_{420}$. The presence of
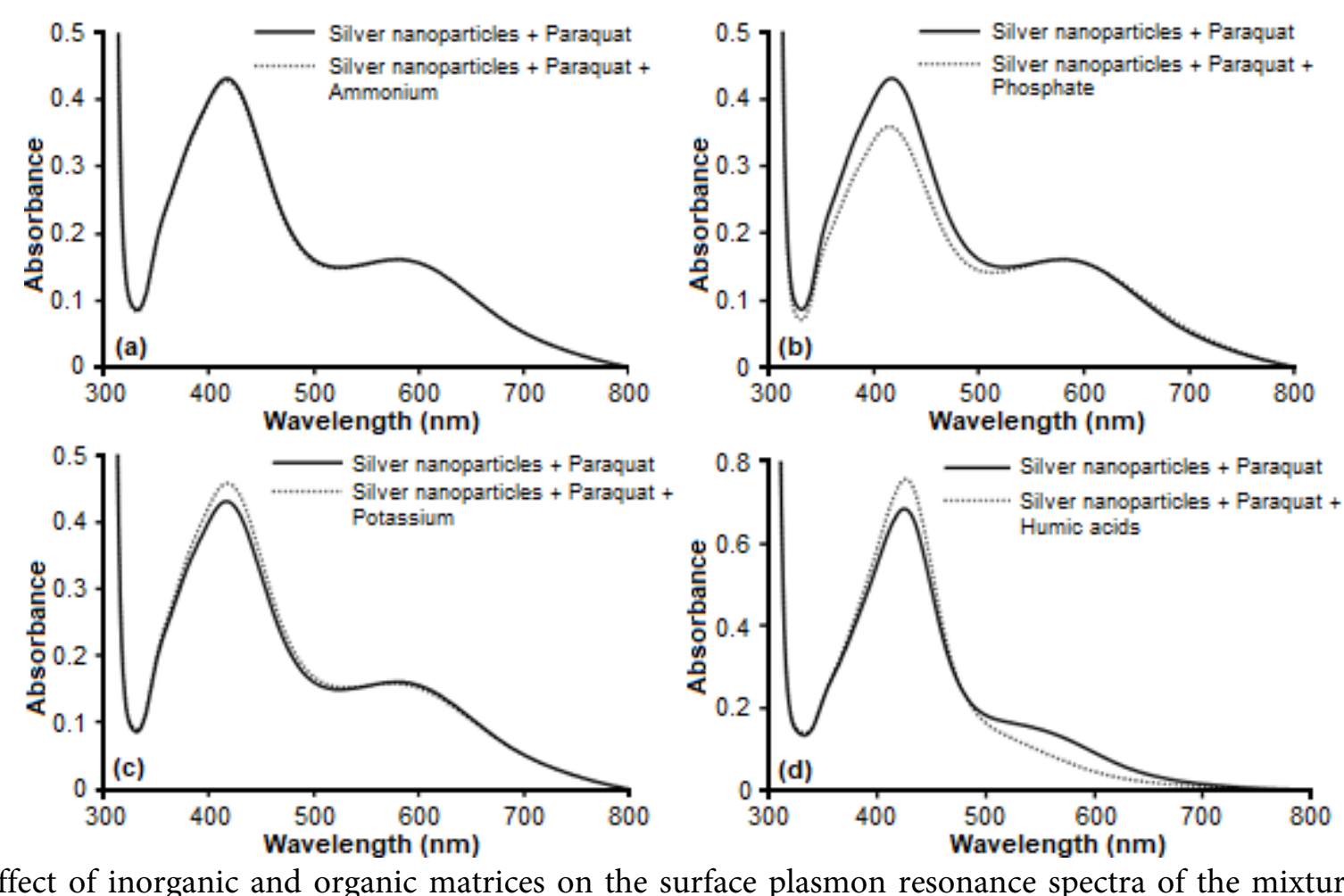

$\mathrm{Na}_{2} \mathrm{HPO}_{4}$ decreased the intensity of the main peak at $420 \mathrm{~nm}$. Because phosphate is in the same group with carboxylic as hard bases[30], it is predicted that phosphate interacted with the surface of silver nanoparticles capped with $p$-hydroxybenzoic acid and replaced the $p$ hydroxybenzoic acid that acts as capping agent on the surface of the silver nanoparticles. This caused the destabilization of silver nanoparticles followed by aggregation. nanoparticles and paraquat changed their spectra. There was an increase in the peak at $420 \mathrm{~nm}$, but the peak at $580 \mathrm{~nm}$ disappeared. These results indicate that humic acids inhibit the aggregation of silver nanoparticles by paraquat. It is predicted that the interaction of paraquat with humic acid was stronger than that of silver nanoparticles so that the aggregation of silver nanoparticles capped with $p$-hydroxybenzoic acid facilitated by paraquat became blocked. Paraquat can interact with humic acid through ionic interactions as well as the formation of charge transfer complex [28].

Fig 6. Effect of inorganic and organic matrices on the surface plasmon resonance spectra of the mixture of silver nanoparticles and paraquat

The presence of humic acid in the mixture of silver 


\section{Comparison with another Method}

The result of the determination of paraquat using silver nanoparticles capped with $p$-hydroxybenzoic acid was compared to another spectrophotometric method [31]. In this method, paraquat is reduced by ascorbic acid in alkaline solution resulting in the blue paraquat radical. The paraquat radical is measured with a UV-visible spectrophotometer at $600 \mathrm{~nm}$.

Four concentrations of standard paraquat ranging from $1.0 \times 10^{-3}$ to $4.0 \times 10^{-3} \mathrm{M}$ were measured by these two methods. A comparison of the result of the determination from different methods can be made by plotting the result in an $x-y$ graph. The confidence interval for the slope and intercept of the line is used to find out whether these two methods produce the same or different results [32]. Our results are presented in Fig. 7. The equation of the line obtained is $\mathrm{y}=0.9105 \mathrm{x}-0.00005(\mathrm{r}=$ 0.9991). The confidence interval for the slope and intercept of the line lies between 0.9278 to 1.1508 and -0.00033 to 0.00025 , respectively. Because the confidence interval for the slope and intercept includes one and zero, it is concluded that the difference of the results obtained using silver nanoparticles capped with $p$-hydroxybenzoic acid and ascorbic acid method is not significant. Because of the instability of the paraquat radical, silver nanoparticles capped with $p$-hydroxybenzoic acid is more suitable for the determination of paraquat.

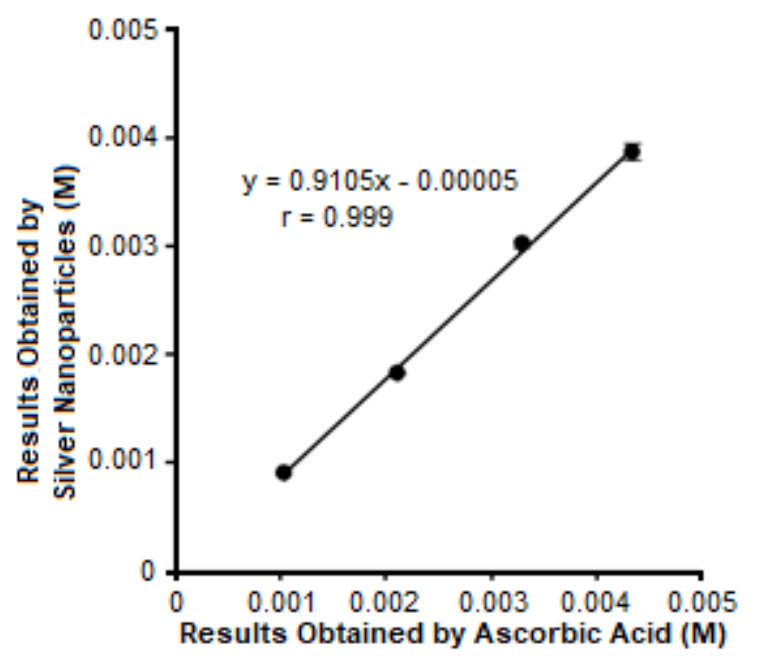

Fig 7. Comparison of the result of the determination obtained using ascorbic acid and silver nanoparticles capped with p-hydroxybenzoic acid

\section{- CONCLUSION}

In this work, a simple method for the determination of paraquat in aqueous medium was developed based on the aggregation of silver nanoparticles capped with $p$-hydroxybenzoic acid. Paraquat acts as a bridge between the nanoparticles and causes the aggregation of nanoparticles followed by the change of the ratio of absorbance obtained at 580 and $420 \mathrm{~nm}$. The absorbance ratio is linearly correlated with the concentration of paraquat. Validation of the developed method indicates that this method could be useful for the colorimetric determination of paraquat in the aqueous medium.

\section{- ACKNOWLEDGMENTS}

This work was supported by the Indonesian Ministry of Research, Technology, and Higher Education through BPPDN Scholarship with contract number: $1251.15 / \mathrm{E} 4.4 / 2013$. The authors would like to thank Philip Anggo Krisbiantoro for providing humic acid. G.G also acknowledges Universitas Tanjungpura for support in writing this paper through the Professional Communication Workshop at Kansas State University.

\section{REFERENCES}

[1] Durán, N., Durán, M., de Jesus, M.B., Seabra, A.B., Fávaro, W.J., and Nakazato, G., 2016, Silver nanoparticles: A new view on mechanistic aspects on antimicrobial activity, Nanomed. Nanotechnol. Biol. Med., 12 (3), 789-799.

[2] Ravindran, A., Elavarasi, M., Prathna, T.C., Raichur, A.M., Chandrasekaran, N., and Mukherjee, A., 2012, Selective colorimetric detection of nanomolar $\mathrm{Cr}(\mathrm{VI})$ in aqueous solutions using unmodified silver nanoparticles, Sens. Actuators, B, 166-167, 365-371.

[3] Wu, X., Xu, Y., Dong, Y., Jiang, X., and Zhu, N., 2013, Colorimetric determination of hexavalent chromium with ascorbic acid capped silver nanoparticles, Anal. Methods, 5 (2), 560-565.

[4] Frost, M.S., Dempsey, M.J., and Whitehead, D.E., 2017, The response of citrate functionalised gold 
and silver nanoparticles to the addition of heavy metal ions, Colloids Surf., A, 518, 15-24.

[5] Lee, P.C., and Meisel, D., 1982, Adsorption and surface-enhanced Raman of dyes on silver and gold sols, J. Phys. Chem., 86 (17), 3391-3395.

[6] Ajitha, B., Reddy, Y.A.K., Reddy, P.S., Jeon, H.J., and Ahn, C.W., 2016, Role of capping agents in controlling silver nanoparticles size, antibacterial activity and potential application as optical hydrogen peroxide sensor, RSC Adv., 6 (42), 36171-36179.

[7] Rycenga, M., Cobley, C.M., Zeng, J., Li, W., Moran, C.H., Zhang, Q., Qin, D., and Xia, Y., 2011, Controlling the synthesis and assembly of silver nanostructures for plasmonic applications, Chem. Rev., 111 (6), 3669-3712.

[8] Ratnarathorn, N., Chailapakul, O., Henry, C.S., and Dungchai, W., 2012, Simple silver nanoparticle colorimetric sensing for copper by paper-based devices, Talanta, 99, 552-557.

[9] Annadhasan, M., Muthukumarasamyvel, T., Sankar Babu, V.R., and Rajendiran, N., 2014, Green synthesized silver and gold nanoparticles for colorimetric detection of $\mathrm{Hg}^{2+}, \mathrm{Pb}^{2+}$, and $\mathrm{Mn}^{2+}$ in an aqueous medium, ACS Sustainable Chem. Eng., 2 (4), 887-896.

[10] Shrivas, K., Sahu, S., Patra, G.K., Jaiswal, N.K., and Shankar, R., 2016, Localized surface plasmon resonance of silver nanoparticles for sensitive colorimetric detection of chromium in surface water, industrial wastewater and vegetable samples, Anal. Methods, 8 (9), 2088-2096.

[11] Roto, R., Marcelina, Aprilita, N.H., Mudasir, Natsir, T.A., and Mellisani, B., 2017, Investigation on the effect of the addition of $\mathrm{Fe}^{3+}$ ion into the colloidal AgNPs in PVA solution and understanding its reaction mechanism, Indones. J. Chem., 17 (3), 439445.

[12] Ma, Y., Niu, H., Zhang, X., and Cai, Y., 2011, Onestep synthesis of silver/dopamine nanoparticles and visual detection of melamine in raw milk, Analyst, 136 (20), 4192-4196.

[13] Dubas, S.T., and Pimpan, V., 2008, Humic acid assisted synthesis of silver nanoparticles and its application to herbicide detection, Mater. Lett., 62 (17-18), 2661-2663.

[14] Lee, P.C., Bordelon, Y., Bronstein, J., and Ritz, B., 2012, Traumatic brain injury, paraquat exposure, and their relationship to Parkinson disease, Neurology, 79 (20), 2061-2066.

[15] Pezzoli, G., and Cereda, E., 2013, Exposure to pesticides or solvents and risk of Parkinson disease, Neurology, 80 (22), 2035-2041.

[16] Takino, M., Daishima, S., and Yamaguchi, K., 2000, Determination of diquat and paraquat in water by liquid chromatography/electrospray-mass spectrometry using volatile ion-pairing reagents, Anal. Sci., 16 (7), 707-711.

[17] Pizzutti, I.R., Vela, G.M.E., de Kok, A., Scholten, J.M., Dias, J.V., Cardoso, C.D., Concenço, G., and Vivian, R., 2016, Determination of paraquat and diquat: LC-MS method optimization and validation, Food Chem., 209, 248-255.

[18] Gusrizal, G., Santosa, S.J., Kunarti, E.S., and Rusdiarso, B., 2018, Two highly stable silver nanoparticles: Surface plasmon resonance spectra study of silver nanoparticles capped with $m$ hydroxybenzoic acid and $p$-hydroxybenzoic acid, Molekul, 13 (1), 30-37.

[19] Gusrizal, 2017, Sintesis nanopartikel perak melalui reduksi ion perak dengan asam 2-, 3-, dan 4hidroksibenzoat serta aplikasinya untuk penentuan parakuat, Dissertation, Department of Chemistry, Universitas Gadjah Mada, Indonesia.

[20] Gusrizal, G., Santosa, S.J., Kunarti, E.S., and Rusdiarso, B., 2016, Dual function of $p$ hydroxybenzoic acid as reducing and capping agent in the rapid and simple formation of stable silver nanoparticles, Int. J. Chemtech Res., 9 (9), 472-482.

[21] Wang, H., Qiao, X., Chen, J., Wang, X., and Ding, S., 2005, Mechanisms of PVP in the preparation of silver nanoparticles, Mater. Chem. Phys., 94 (2-3), 449-453.

[22] Yang, J., Yin, H., Jia, J., and Wei, Y., 2011, Facile synthesis of high-concentration, stable aqueous dispersions of uniform silver nanoparticles using aniline as a reductant, Langmuir, 27 (8), 5047-5053. 
[23] Khodashenas, B., and Ghorbani, H.R., 2019, Synthesis of silver nanoparticles with different shapes, Arabian J. Chem., 12 (8), 1823-1838.

[24] Wei, H., Abtahi, S.M.H., and Vikesland, P.J., 2015, Plasmonic colorimetric and SERS sensors for environmental analysis, Environ. Sci.: Nano, 2 (2), 120-135.

[25] Jin, W., Huang, P., Wu, F., and Ma, L.H., 2015, Ultrasensitive colorimetric assay of cadmium ion based on silver nanoparticles functionalized with 5sulfosalicylic acid for wide practical applications, Analyst, 140 (10), 3507-3513.

[26] Pinto, V.V., Ferreira, M.J., Silva, R., Santos, H.A., Silva, F., and Pereira, C.M., 2010, Long time effect on the stability of silver nanoparticles in aqueous medium: Effect of the synthesis and storage conditions, Colloids Surf., A, 364 (1-3), 19-25.

[27] Senesi, N., D’Orazio, V., and Miano, T.M., 1995, Adsorption mechanisms of s-triazine and bipyridylium herbicides on humic acids from hop field soils, Geoderma, 66 (3-4), 273-283.
[28] Roldán, M.L., Corrado, G., Francioso, O., and Sanchez-Cortes, S., 2011, Interaction of soil humic acids with herbicide paraquat analyzed by surfaceenhanced Raman scattering and fluorescence spectroscopy on silver plasmonic nanoparticles, Anal. Chim. Acta, 699 (1), 87-95.

[29] Vilela, D., González, M.C., and Escarpa, A., 2012, Sensing colorimetric approaches based on gold and silver nanoparticles aggregation: Chemical creativity behind the assay. A review, Anal. Chim. Acta, 751, 24-43.

[30] Pearson, R.G., 1968, Hard and soft acid and bases, HSAB, Part I: Fundamental principles, J. Chem. Educ., 45 (9), 581-587.

[31] Shivhare, P., and Gupta, V.K., 1991, Spectrophotometric method for the determination of paraquat in water, grain and plant materials, Analyst, 116 (4), 391-393.

[32] Miller, J.N., and Miller, J.C., 2010, Statistics and Chemometrics for Analytical Chemistry, $6^{\text {th }}$ Ed., Pearson Education, Canada. 